\title{
Scavenging the hydroxyl radical by 2,2-diphenyl-1-picrylhydrazyl
}

\author{
Elena N. Hristea ${ }^{\text {a }}$ Mihaela Hillebrand, ${ }^{\mathrm{b}}$ Miron T. Caproiu, ${ }^{\mathrm{c}}$ Horia Caldararu, ${ }^{\mathrm{a}}$ Titus \\ Constantinescu, ${ }^{a}$ and Alexandru T. Balaban ${ }^{\mathrm{d}, *}$
}

a Institute of Physical Chemistry "I. G. Murgulescu” of the Romanian Academy, Spl. Independentei 202, 77208 Bucharest, Romania, ${ }^{b}$ University of Bucharest, Department of Physical Chemistry, Bulevardul Carol I No. 13, Bucharest, Romania, ${ }^{c}$ Institute of Organic Chemistry “C. D. Nenitzescu” of the Romanian Academy, Spl. Independentei 202B, Bucharest 15-256, Romania, ' Texas A\&M University, Department of Marine Sciences, 5007 Avenue U, Galveston, TX 77551, USA

E-mail: balabana@tamug.tamu.edu

\section{Dedicated to Professor C. D. Nenitzescu on the $100^{\text {th }}$ anniversary of his birth} (received 23 Aug 2001; accepted 24 Jun 2002; published on the web 02 Jul 2002)

\begin{abstract}
The reaction between 2,2-diphenyl-1-picrylhydrazyl (DPPH) and the hydroxyl free radical generated either photochemically or thermally leads to a mixture of 2-(4-hydroxyphenyl)-2phenyl-1-picrylhydrazine (3) and 2-(4-nitrophenyl)-2-phenyl-1-picrylhydrazine (5). Proof for the structure of 3 was obtained from NMR spectra, methylation to the previously known 2-(4anisyl)-2-phenyl-1-picrylhydrazine, and oxidation to the 2-(4-hydroxyphenyl)-2-phenyl-1picrylhydrazyl free radical.
\end{abstract}

Keywords: 2,2-Diphenyl-1-picrylhydrazyl, hydroxyl radical, 2-(4-hydroxyphenyl)-2-phenyl-1picrylhydrazine, 2-(4-hydroxyphenyl)-2-phenyl-1-picrylhydrazyl, 2-(4-anisyl)-2-phenyl-1picrylhydrazine

\section{Introduction}

The literature contains contradicting statements on the reaction between 2,2-diphenyl-1picrylhydrazyl (DPPH, 1) and reactive free radicals. The picryl group is abbreviated as Pic. In most cases, the reaction is a homolytic substitution of a hydrogen atom in a para-position of one of the phenyl rings. ${ }^{1-9}$ thus nitrogen dioxide affords 2-(4-nitrophenyl)-2-phenyl-1picrylhydrazine (5) and/or the corresponding free radical (6). In one case, a different reaction occurs, namely an ipso-substitution of a 4-nitro group in the picryl ring. ${ }^{5}$ Older data mention, however, that some free radicals ${ }^{8}$ such as hydroxyl may become attached to the 1-nitrogen atom 
yielding the diamagnetic species 2,2-diphenyl-1-hydroxy-1-picrylhydrazine. ${ }^{10}$ In the present paper we correct this statement, proving that the reaction product is $\mathbf{3}$, involving the homolytic attack of one of the phenyl rings.

We have studied the reaction between DPPH and the hydroxyl radical generated by different reactions, namely: the photochemical decomposition of: (a) 2-mercaptopyridin- $\mathrm{N}$-oxide (FotoFenton ${ }^{\mathrm{TM}} 1$ reagent); $;^{1,11-13}$ (b) ortho-hydroxy-acetophenoneoxime (FotoFenton ${ }^{\mathrm{TM}} 2$ ); ${ }^{1,14}$ and (c) salicylaldoxime; ${ }^{14}$ thermal decomposition of: (d) tert-butyl hydroperoxide or cumyl hydroperoxide; (e) the Fenton reagent (hydrogen peroxide plus ferrous salts); and (f) the supramolecular complex $[18 \mathrm{C} 6 \ldots \mathrm{K}]^{+} \mathrm{HO}^{-}$where $18 \mathrm{C} 6$ is the abbreviation for the 18-crown-6 ether. ${ }^{15}$ Three of these reactions, namely (b), (c), and (f), led to the formation of 2-(4hydroxyphenyl)-2-phenyl-1-picrylhydrazine 3 along with a para-nitration product (5) of DPPH. In the other three cases the complex reaction mixture did not contain 3.

Reaction products were isolated by preparative TLC, and the structure of $\mathbf{3}$ was confirmed by NMR spectra, by oxidation to a free hydrazylic radical 4 , and by methylation of the hydroxyl group with methyl iodide yielding the corresponding methoxy derivative $\mathbf{7}$ that had been obtained earlier via a different route. ${ }^{16}$

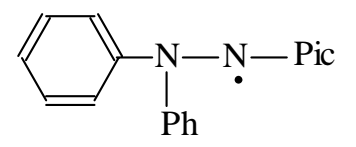

1

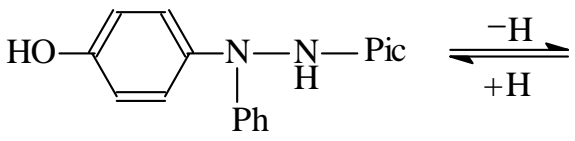

3

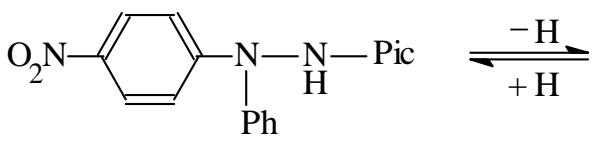

5

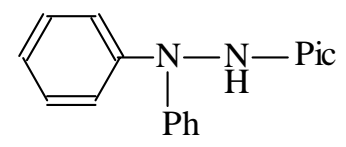

2

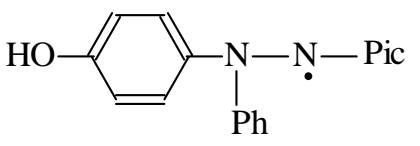

4

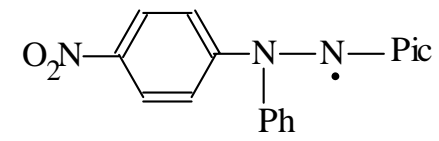

6

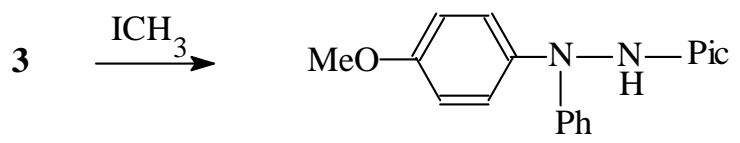

7

Electronic absorption spectra allowed to follow the disappearance of DPPH and the formation of reaction products, as will be shown below. 


\section{Reaction between DPPH and the hydroxyl radical}

Photochemical reaction (b) between DPPH and ortho-hydroxy-acetophenoneoxime (FotoFenton $^{\mathrm{TM}_{2}}$ reagent), 8a. Both ortho-hydroxy-acetophenoneoxime (8a) and salicylaldoxime (8b) can be split photochemically affording hydroxyl radicals. Experiments carried out with DPPH and each of these reagents have shown that only 8a leads to significant yields of an adduct between DPPH and a hydroxyl radical; additionally, a para-nitration product (5) of DPPH was isolated in comparable yield. By contrast, when $\mathbf{8 b}$ was employed in reaction (c), the yield of $\mathbf{3}$ was insignificant (much lower than that of 5).

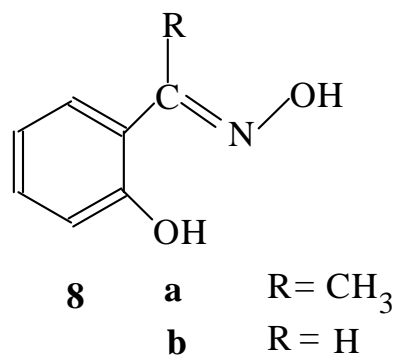

We generated photochemically HO radicals from 8a by UV irradiation in benzene at room temperature, starting with an equimolar ratio of 8a and $\mathrm{DPPH}$, under efficient stirring. The reaction products were isolated after concentration by preparative TLC, using dichloromethane for elution. Solid ascorbic acid was added during the evaporation of dichloromethane under reduced pressure at room temperature for avoiding oxidations. Visually, during the first 15 minutes of UV irradiation one observes how the violet color of the DPPH solution changes into deep cherry-red. The main constituents of the reaction mixture after 90 minutes were: 2,2diphenyl-1-picrylhydrazine (2, 45\% yield), 2-(4-hydroxyphenyl)-2-phenyl-1-picrylhydrazine (3, $12 \%$ yield), and 2-(4-nitrophenyl)-2-phenyl-1-picrylhydrazine (5, 18\% yield).

In Figure 1 one can observe how the visible absorption spectrum changed during the reaction: the DPPH peaks with $\lambda_{\max } 328$ and $520 \mathrm{~nm}$ decreased, and new bands appeared due to the formation of reaction products 2 (316 nm), ${ }^{17,18} \mathbf{3}$ (304, 424, and $\left.556 \mathrm{~nm}\right)$ and 5 (324, 344, and $372 \mathrm{~nm}){ }^{19}$ Isosbestic points at 304, 356, and $424 \mathrm{~nm}$ can be observed. 


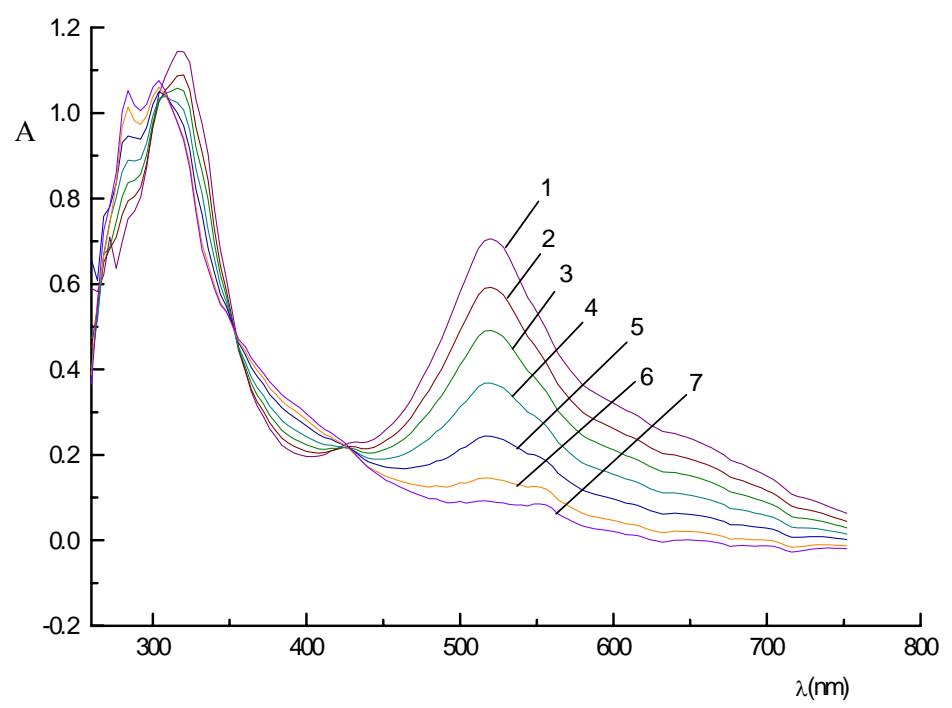

Figure 1. Electronic absorption spectrum of the reaction mixture obtained by irradiating an equimolar mixture of DPPH and 8a in benzene after various irradiation times (minutes): $1=$ after 5 minutes; 2 = 15 min.; 3 = 25 min.; 4 = 35 min.; $5=45$ min.; $6=55$ min.; $7=65$ min.

We checked that UV irradiation of $\mathbf{8 a}$ or $\mathbf{8 b}$ in benzene generates $\mathrm{HO}$ radicals by trapping them with tert-butyl-phenylnitrone, 9: the resulting nitroxide $\mathbf{1 0}$ had in both cases ESR hyperfine constants that were consistent with literature data: $\mathrm{a}_{\mathrm{N}}=14.6 \mathrm{G}$ and $\mathrm{a}_{\mathrm{H}}=2.35 \mathrm{G}^{20}$

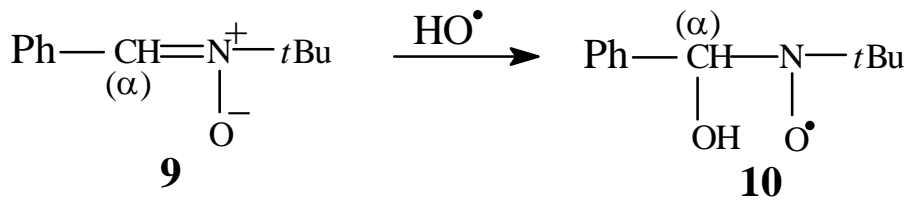

Previous experimental evidence showed that also in other homolytic processes DPPH underwent disproportionation affording 2-(4-nitrophenyl)-2-phenyl-1-picrylhydrazine (5) along with other products: example are the reaction with bromine, ${ }^{2,6}$ or with acetyl nitrate. ${ }^{19,21,22}$ It is known that oximes such as $\mathbf{8 a}$ and $\mathbf{8 b}$ undergo photolysis of the $\mathrm{N}-\mathrm{O}$ bond generating hydroxyl radicals. $^{22,23}$

In a control reaction, UV irradiation of DPPH alone in benzene does not afford 2, 3, or 5. On irradiating a mixture of $\mathbf{8 a}$ and $\mathrm{DPPH}_{2}$ (2) one obtains only $\mathbf{3}$ with unreacted 2, and no 5. Irradiation of a mixture of $8 \mathrm{a}$ and the crown ether potassium salt of $\mathrm{DPPH},\left[18 \mathrm{C} 6 \ldots \mathrm{K}^{+} \mathrm{DPPH}^{-}\right.$, one obtains both 3 and 5 . 
Photochemical reaction (a) between DPPH and 2-mercaptopyridin- $N$-oxide (FotoFenton ${ }^{\mathrm{TM}} 1$ reagent). On irradiating similarly the mixture of these two compounds in benzene, compound 3 could not be detected; however, the nitro-derivative $\mathbf{5}$ was formed.

Reaction (e) between DPPH and Fenton's reagent. The reaction of DPPH with ferrous sulfate and hydrogen peroxide was carried out in two variants at room temperature with efficient stirring: (i) in a biphasic liquid system (water - methylene chloride) and (ii) in homogeneous acetonitrile solution. In both variants we could not detect by TLC any formation of compound $\mathbf{3}$, but only traces of 5 .

Reaction (d) between DPPH and alkyl hydroperoxides. It had been reported that DPPH reacts with tert-butyl hydroperoxide affording 2,2-diphenyl-1-hydroxy-1-picrylhydrazine. ${ }^{10}$ On repeating this reaction in benzene at room temperature according to literature data, ${ }^{10}$ we detected 5 (about 40\% yield relative to DPPH), but neither 2,2-diphenyl-1-hydroxy-1-picrylhydrazine, 3, nor its corresponding free radical, 4. Similar results were obtained with cumyl hydroperoxide.

Reaction (f) between DPPH and potassium hydroxide in the presence of crown ether 18C6. In a previous paper ${ }^{15}$ we had shown that in solid/liquid $\left(\mathrm{KOH} / \mathrm{CH}_{2} \mathrm{Cl}_{2}\right)$ or liquid/liquid biphasic systems (aqueous $\mathrm{KOH}$ solution/ $\mathrm{CH}_{2} \mathrm{Cl}_{2}$ ) the free radical DPPH afforded a mixture of products containing 5. On examining again this mixture, we established that it contains also 3 . The mechanism leading to these products is described by the following equations, in which the subscripts s, w, and o stand for solid, water, and organic phase, respectively.

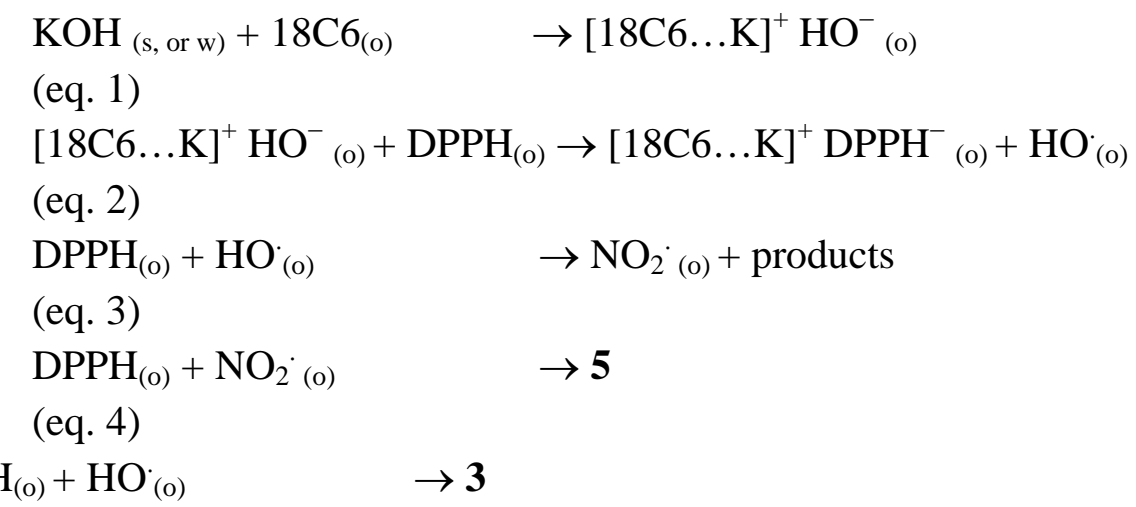

A few comments must be added about these equations. The naked hydroxyl anion forms with $\mathrm{DPPH}$ a salt according to eq. 1. The redox reaction between the free radical DPPH and the hydroxyl anion described in eq. 2 generates the hydroxyl radical that is responsible for the formation (eq. 5) of $\mathbf{3}$ by homolytic substitution of a phenyl ring of DPPH (followed, of course, by a hydrogen transfer from the resulted para-quinonoid structure to the hydrazinic nitrogen yielding 3). At the same time, the hydroxyl radical also initiates a more complicated chain of events (equations 3 and 4) resulting in the formation of 5 along with unidentified products

Properties of 2-(4-hydroxyphenyl)-2-phenyl-1-picrylhydrazine, 3

Compound 3 is a yellow-orange compound with $\lambda_{\max }=304,424$ (shoulder), and $556 \mathrm{~nm}$. Because of its autoxidation in air, $\mathbf{3}$ has to be stabilized by ascorbic acid and therefore we cannot 
report its m.p. or its elemental analysis. However, it was characterized by NMR spectra, its methylation to a known compound and the formation of its corresponding free radical: on oxidation with solid potassium permanganate, compound $\mathbf{3}$ forms a stable violet-colored hydrazylic free radical 4, whose ESR spectrum in benzene has five broad lines similarly to DPPH, with an averaged $\mathrm{a}_{\mathrm{N}}$ hyperfine coupling constant of 8.9 Gauss. Further oxidation affords a bright-red compound with probable structure 11; this compound does not migrate in TLC on silica gel with toluene as mobile phase $\left(\mathrm{R}_{\mathrm{f}}=0\right)$ owing to its amphionic (betainic) structure, unlike DPPH $\left(\mathrm{R}_{\mathrm{f}}=0.60\right)$ or compound $3\left(\mathrm{R}_{\mathrm{f}}=0.53\right)$. Compound 11 is formed from $\mathbf{3}$ in air in the presence of light, therefore one needs ascorbic acid to protect 3 against autoxidation.

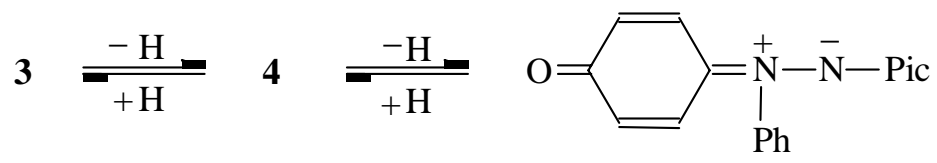

11

In the presence of powdered solid potassium hydroxide, compound 3 can be methylated with methyl iodide affording the methoxy derivative $\mathbf{7}$ described earlier and obtained via a different route. ${ }^{16}$ A solution of $\mathbf{7}$ in methylene chloride can be oxidized by potassium permanganate to the stable 2-(4-methoxyphenyl)-2-phenyl-1-picrylhydrazyl free radical, with two averaged $\mathrm{a}_{\mathrm{N}}$ hfc's = 8.8 Gauss. $^{16}$

It is interesting to speculate about the rich possibilities offered by considering the similarity between an oxygen atom and the enhanced electronegativity of an N-picryl group relative to an aminic NH group. If one considers nitrobenzene as the starting point in such a series of "picrylogs" and, in addition, the possible "para-phenylogs" (avoiding ortho-phenylogs for steric reasons), then one obtains Figure 2. We have only started to explore these possibilities having prepared betaines contained in this chart. One should also consider that the three hydrazylic structures on the left-hand side of this chart can give rise to stable free radicals, and that somewhat less stable free radicals are also obtained from their phenylogs. 

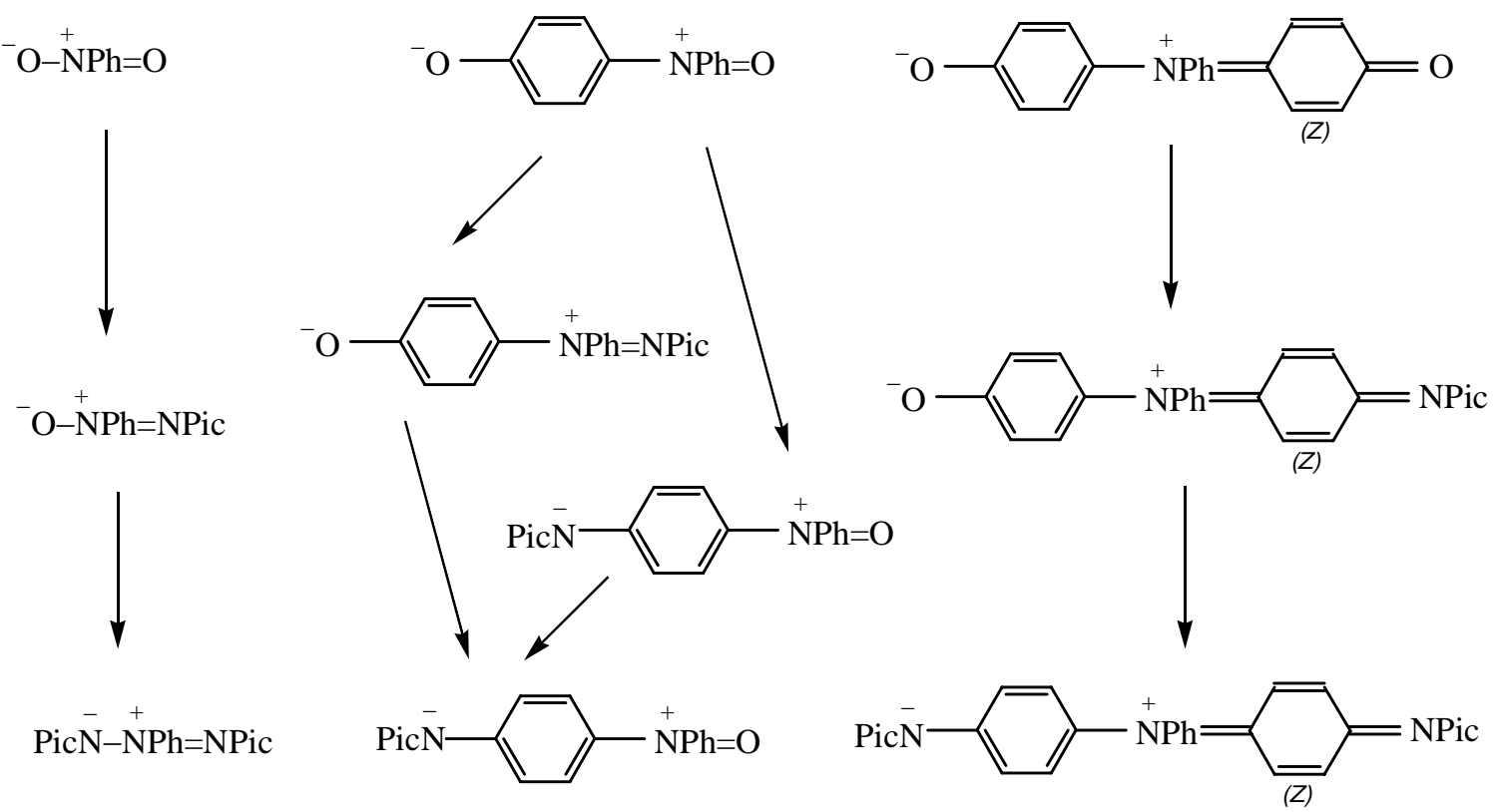

Figure 2. The chart of "picrylogs" of nitrobenzene on different levels (linked by arrows on replacing one or both oxygen atoms by N-picryl groups), and "phenylogs" on the same level in three different columns (replacement of covalent bond(s) by one or two para-phenylene groups). It should be noted that for each formula one should also consider a second main resonance structure with the negative charge located on the right-hand-side end group.

\section{Conclusions}

From the reaction of DPPH with the hydroxyl radical generated either thermally or photochemically, we obtained a mixture of 2-(4-hydroxyphenyl)-2-phenyl-1-picrylhydrazine (3) and 2-(4-nitrophenyl)-2-phenyl-1-picrylhydrazine (5). Thus, it was proved that DPPH could act as a scavenger for the hydroxyl radical and that the reaction involved a homolytic substitution of one of the phenyl rings of DPPH yielding 3. The second product 5 is formed, as in other reactions of DPPH that we described earlier, via a series of secondary processes.

\section{Experimental Section}

General Procedures. Compounds 1 (DPPH, Fluka), 9 (tert-butyl-phenylnitrone, Fluka), FotoFenton-1 (Molecular Probes), 8a (FotoFenton-2, Molecular Probes), 8b (salicylaldoxime, Fluka), tert-butylhydroperoxide (Fluka), cumylhydroperoxide (Fluka), and 18C6 (Merck), and were of analytical purity. We prepared as described earlier 2-(4-nitrophenyl)-2-phenyl-1picrylhydrazine (5), $\mathrm{DPPH}_{2}(2)$ and the supramolecular complex $[18 \mathrm{C} 6 . . \mathrm{K}]^{+} \mathrm{DPPH}^{-}{ }^{18}$ 
Electronic absorption spectra were recorded with a Unicam-UV-VIS spectrophotometer using Vision Software V3.33, and ESR spectra with a Jeol JES-3B instrument at $100 \mathrm{kHz}$ and modulation using X-band frequency. 1H-NMR spectra were recorded with a Varian Gemini 300 BB using TMS as internal standard.

\section{Formation of 2-(4-hydroxyphenyl)-2-phenyl-1-picrylhydrazine (3)}

(i) Photochemically from DPPH and 8a, (reaction b). An equimolar mixture of DPPH (1) and 8a ( $0.5 \mathrm{~g}$ of mixture) in $8 \mathrm{~mL}$ benzene was irradiated in a quartz vial with a tungsten lamp (500 $\mathrm{W}$ ) at room temperature with magnetic stirring for 90 minutes. The initially violet solution became cherry-red. It was concentrated under vacuum and purified by preparative TLC (silica gel Merck GF 254, toluene, three times). The yellow-orange band with $\mathrm{R}_{\mathrm{f}}=0.53$ (below the unreacted DPPH with $\mathrm{R}_{\mathrm{f}}=0.60$ ) was eluted with dichloromethane. Into this solution a known small amount of solid ascorbic acid was added, and the product was evaporated at room temperature (yield of 3 about $12 \%$ ). Its ${ }^{1} \mathrm{H}-\mathrm{NMR}$ spectrum was determined in $\mathrm{CDCl}_{3}$, which dissolves only 3 leaving the solid ascorbic acid.

${ }^{1} \mathrm{H}-\mathrm{NMR}\left(\mathrm{CDCl}_{3}, \delta \mathrm{ppm}, \mathrm{J} \mathrm{Hz}\right.$ ): 10.88 (broad-s, 1H, OH, deuterable); 10.09 (bs, 1H, NH, deuterable); 7.20 (d, 2H, H-8 \& H-12, 9.3 Hz); 7.15 (d, 2H, H-9 \& H-11, 9.3 Hz); 7.07 (dd, 2H, $\mathrm{H}-14$ \& H-18, 1.3 \& $8.3 \mathrm{~Hz}$ ); 7.38-7.30 (m, 3H, H-16, H-17, H-18).

${ }^{13} \mathrm{C}-\mathrm{NMR}\left(\mathrm{CDCl}_{3}, \delta \mathrm{ppm}\right): 157.84$ (C-10); 146.37 (C-13); 142.03 (C-1); 141.42 (C-7); 136.58 (C-13); 140.0 (broad, 2C, C-2 \& C-6); 128.22 (CH-16); 126.23 (b, CH-3/5); 125.01 (b, CH-5/3); $129.48(2 \mathrm{CH}) ; 119.02(2 \mathrm{CH}) ; 116.19(2 \mathrm{CH})$.

The second product, 2-(4-nitrophenyl)-2-phenyl-1-picrylhydrazine (5), was isolated similarly from the TLC experiment and the solvent (dichloromethane) was removed (rotavapor): 18\% yield. Its identity was confirmed by comparison with an authentic specimen ${ }^{3,6,19}$ via TLC and NMR data.

(ii) Photochemically from DPPH and 8b (reaction c). Proceeding similarly with DPPH and salicylaldoxime, compound 3 was isolated only in traces.

(iii) Thermally from DPPH and the supramolecular complex $\left[18 \mathrm{C6} . . . \mathrm{K}^{+} \mathrm{OH}^{-}\right.$, (reaction $\mathrm{f}$ ). Under the conditions indicated earlier, ${ }^{15} \mathrm{DPPH}$ in benzene and the solid supramolecular complex [18C6...K $]^{+} \mathrm{OH}^{-}$reacted forming the same mixture of $\mathbf{3}$ and $\mathbf{5}$ as in the photochemical reaction between DPPH and 8a. The purification was carried out as indicated above, by preparative TLC on silica gel Merck GF 254, toluene, three times.

Preparation of 2-(4-anisyl)-2-phenyl-1-picrylhydrazine (7). Compound 3 dissolved in methylene chloride and admixed with solid ascorbic acid was treated at room temperature for one month with methyl iodide and the supramolecular complex $[18 \mathrm{C} 6 \ldots \mathrm{K}]^{+} \mathrm{OH}^{-}$(previously prepared from 18C6 and potassium hydroxide). ${ }^{16}$ After washing the mixture three times with $1 \mathrm{~N}$ hydrochloric acid, the solution was dried on sodium sulfate, the methylene chloride was evaporated (rotavapor) and the product was isolated by preparative TLC, proving to be identical with an authentic sample described earlier. ${ }^{16}$ Oxidation with potassium permanganate afforded the same stable free radical 2-(4-anisyl)-2-phenyl-1-picrylhydrazyl that was described earlier. ${ }^{16}$ 
${ }^{1} \mathrm{H}-\mathrm{NMR}\left(\mathrm{CDCl}_{3}, \delta\right.$ ppm, J Hz): 10.04 (bs, 1H, NH, deuterable); 9.21 (bs, 1H, H3/H5); 8.53 (bs, 1H, H-5/3); 6.88 (d, 2H, H9 \& H11, 9.1 Hz); 7,32 (t, 2H, H-15 \& H-17, 7.5 Hz); 7.17 (tt, 1H, H16, 1.6 \& 7.5 Hz); 7.11 (d, 2H, H-8 \& H-12, 9.1 Hz); 7.01 (dd, 2H, H-14 \& H-18, 1.6 \& 7.5 Hz); 3.80 (s, 3H, OMe).

Control experiments. No evidence for the formation of 3 was obtained in the following control experiments: (i) photochemical UV irradiation of $\mathrm{DPPH}_{2}$ (2) with 8a, when 2 was recovered; (ii) photochemical UV irradiation of the supramolecular complex $[18 \mathrm{C} 6 . . \mathrm{K}]^{+} \mathrm{DPPH}^{-}$with 8a, when after neutralization with hydrochloric acid only the corresponding $\mathrm{DPPH}_{2}$ (2) was detected, along with 5 that was formed in 35\% yield; (iii) reactions of DPPH with alkyl hydroperoxides at temperatures $30-50^{\circ} \mathrm{C}$ for 24 hrs, when only 5 was formed; photochemical reaction of DPPH with 2-mercaptopyridin-N-oxide (FotoFenton ${ }^{\mathrm{TM}} 1$ reagent) obtained from its sodium salt, ${ }^{13}$ when only the formation of $\mathbf{5}$ was detected; (iv) reaction of DPPH with Fenton's reagent, either in homogeneous acetonitrile/water 2:1 v/v solution, or in a heterogeneous benzene/water system, when only the formation of $\mathbf{2}$ and $\mathbf{5}$ was detected.

Oxidation of 3 to the 2-(4-hydroxyphenyl)-2-phenyl-1-picrylhydrazyl free radical (4) and the betaine (11). After extracting compound 3 with methylene chloride from the mixture with ascorbic acid, solid potassium permanganate was added, when a violet solution resulted. The degassed solution showed a five-line ESR spectrum with two averaged hfc's $\mathrm{a}_{\mathrm{N}}=8.8$ Gauss. On longer standing over $\mathrm{KMnO}_{4}$, the solution became bright-red, and TLC on silica gel Merck GF 254 (toluene) showed that a compound with probable structure 11 that had $R_{f}=0$ was formed. Its reduction with ascorbic acid formed back 3.

\section{Acknowledgements}

We express our thanks Molecular Probes for samples of FotoFenton ${ }^{\mathrm{TM}} 1$ and FotoFenton ${ }^{\mathrm{TM}} 2$.

\section{References}

1. Forrester, A. R.; Hay, J. M.; Thomson, R. H. Organic Chemistry of Stable Free Radicals, Academic Press: London, 1968; p 137.

2. Currie, P. F.; Quail, J. W.; Weil, J. A. Can. J. Chem. 1980, 58, 723.

3. Weil, J. A.; Sane, K. V.; Kinkade, J. M. J. Phys. Chem. 1961, 65, 710.

4. Chen, M. M.; Sane, K. V.; Walter, R. I.; Weil, J. A. J. Phys. Chem. 1961, 65, 713.

5. Flood, J.; Russell, K. E. Can. J. Chem. 1975, 53, 1123.

6. Currie, P. F.; Quail, J. W.; Rusk, A. C. M.; Weil, J. A. Can. J. Chem. 1983, 61, 1760.

7. Gille, I.; Prosch, T.; Stoesser, R. Radiat. Phys. Chem. 1992, 40, 461.

8. Kemp, T. J. Progress in Reaction Kinetics and Mechanism 1999, 24, 287. 
9. Constantinescu, T.; Caproiu, M. T.; Zarna, N.; Caragheorgheopol, A.; Caldararu, H.; Stanciuc, G.; Radu, M.; Badescu V.; Balaban, A. T. New J. Chem. 1997, 21, 575.

10. Dulog, L.; Baum, G. Chem. Ber. 1971, 104, 661.

11. Haugland, R. P. Handbook of Fluorescent Probes and Research Chemicals, $6^{\text {th }}$ Edn; Netherlands, 1996; p 485.

12. Boiven, J.; Crepon, E.; Zard, S. Z. Tetrahedron Lett. 1990, 31, 6869.

13. Barton, D. H. R.; Bridon, D.; Fernandez-Picot, I.; Zard, S. Z. Tetrahedron Lett. 1987, 43, 2733.

14. Grellmann, K. H.; Tauer, E. Tetrahedron Lett. 1974, 42, 3707.

15. Luca, C.; Ionita, P.; Constantinescu, T.; Caldararu, H.; Caragheorgheopol, A.; Caproiu, M. T. Rev. Roum. Chim. 1997, 42, 985.

16. Covaci, I. C.; Constantinescu, T.; Caproiu, M. T.; Caldararu, H.; Ionita, P.; Balaban, A. T. Polish J. Chem. 2001, 75, 1427.

17. Weil, J. A.; Janusonis, G. A. J. Org. Chem. 1962, 27, 1248.

18. Luca, C.; Ionita, P.; Constantinescu, T. Rev. Roum. Chim. 1994, 39, 1141.

19. Ionita, P.; Caproiu, M. T.; Luca, C.; Constantinescu, T.; Caldararu, H.; Balaban, A. T. J. Labelled Comp. Radiopharm. 1998, 51, 791.

20. Buettner, G. R. Free Rad. Biol. Med. 1987, 3, 259.

21. Konior, R. J.; Walter, R. I.; Bologa, U. L.; Caproiu, M.T.; Negoita, N.; Balaban A.T. Polish J. Chem. 1994, 68, 2451.

22. Mc-Carroll, A. J.; Walton, J. C. J. Chem. Soc., Perkin Trans. 2 2000, 2399.

23. Damiani, E.; Carloni, P.; Stipa, P.; Greci, L. Free Rad. Res. 1999, 31, 113. 